\title{
Novel Higgsino dark matter signal interpretation at the LHC
}

\author{
Ernesto Arganda $\odot,{ }^{1,2, *}$ Antonio Delgado $\odot,{ }^{3, \dagger}$ Roberto A. Morales $\odot,{ }^{1, \hbar}$ and Mariano Quirós $\oplus^{4, \S}$ \\ ${ }^{1}$ Instituto de Física Teórica UAM/CSIC, C/ Nicolás Cabrera 13-15, \\ Campus de Cantoblanco, 28049 Madrid, Spain \\ ${ }^{2}$ IFLP, CONICET-Dpto. de Física, Universidad Nacional de La Plata, \\ C.C. 67, 1900 La Plata, Argentina \\ ${ }^{3}$ Department of Physics, University of Notre Dame, 225 Nieuwland Hall Notre Dame, Indiana 46556, USA \\ ${ }^{4}$ Institut de Física d'Altes Energies (IFAE) and BIST, Campus UAB 08193, Bellaterra, Barcelona, Spain
}

(Received 4 May 2021; accepted 3 August 2021; published 7 September 2021)

\begin{abstract}
In the LHC searches for gluinos it is usually assumed that they decay predominantly into the lightest neutralino plus jets. In this work we perform a proof-of-concept collider analysis of a novel supersymmetric signal in which gluinos decay mostly into jets and the bino-like neutralino $\left(\tilde{\chi}_{3}^{0}\right)$, which in turn decays into the lightest Higgsino-like neutralino $\left(\tilde{\chi}_{1}^{0}\right)$, considered the dark matter candidate, together with the SM-like Higgs boson $(h)$. This new physics signal then consists of an LHC final state made up by four light jets, four $b$-jets, and a large amount of missing transverse energy. We identify $t \bar{t}$, $V+$ jets $(V=W, Z)$, and $t \bar{t}+X\left(X=W, Z, \gamma^{*}, h\right)$ productions as the most problematic backgrounds, and develop a search strategy for the high luminosity phase of the LHC, reaching signal significances at the evidence level for a luminosity of $1000 \mathrm{fb}^{-1}$. The prospects for a luminosity of $3000 \mathrm{fb}^{-1}$ are even more promising, with discovery-level significances.
\end{abstract}

DOI: 10.1103/PhysRevD.104.055003

\section{INTRODUCTION}

After the Higgs boson discovery [1,2] at the LHC lots of efforts of the CMS and ATLAS collaborations are in searches for physics beyond the Standard Model (BSM). So far the results have been null so bounds are put in popular models albeit there are caveats on those bounds. The reinterpretation of the searches are normally done in the context of simplified models where it is easier to draw conclusions. An example of those situations are gluino searches done at the LHC (for a recent summary, see, for instance, $[3,4])$. In most of the cases it is assumed that the gluino decays with a branching ratio equal to 1 to the lightest neutralino plus jets, which in fact makes an implicit assumption on the supersymmetric (SUSY) spectrum and couplings. If this assumption is not fulfilled many experimental bounds could be evaded. It is thus interesting to explore other (less conventional) possibilities, as very often

\footnotetext{
*ernesto.arganda@csic.es † adelgad2@nd.edu

†robertoa.morales@uam.es

§quiros@ifae.es
}

Published by the American Physical Society under the terms of the Creative Commons Attribution 4.0 International license. Further distribution of this work must maintain attribution to the author(s) and the published article's title, journal citation, and DOI. Funded by SCOAP. they are theoretically well motivated, as it is the case we will explore in this paper.

In this work we develop a search strategy for a novel interpretation of Higgsino dark matter signals at the LHC, proposed in [5], where the gluino will not decay predominately to the lightest neutralino plus jets. Although the signature was already studied in previous experimental searches [6-9], the assumed spectrum is different than ours (in most cases the LSP is massless and/or other SUSY cascades are treated) demanding a novel interpretation of this type of signatures. Under very general conditions, that will be explained in Sec. II, there could be several electroweakinos lighter than the gluino, which will change dramatically the signatures at the LHC. The aim of our analysis is more to give a proof of principle, than providing an elaborated strategy, to show which kinematical variables and cuts may be effective for this kind of scenarios. Let us finally emphasize that, in general, it is very important for the next run of the LHC to go beyond the usual simplified models, and to design searches, to look for kinematic variables and to optimize cuts, to be sensitive to more scenarios than just the ones captured by simplified models or spectra.

The rest of the paper is organized as follows. The general theoretical framework for the model we will consider is provided in Sec. II. In this framework our guideline will be the possibility of having a $1.1 \mathrm{TeV}$ Higgsino as dark matter. The collider analysis will be done in Sec. III while our conclusions will be drawn in Sec. IV. 

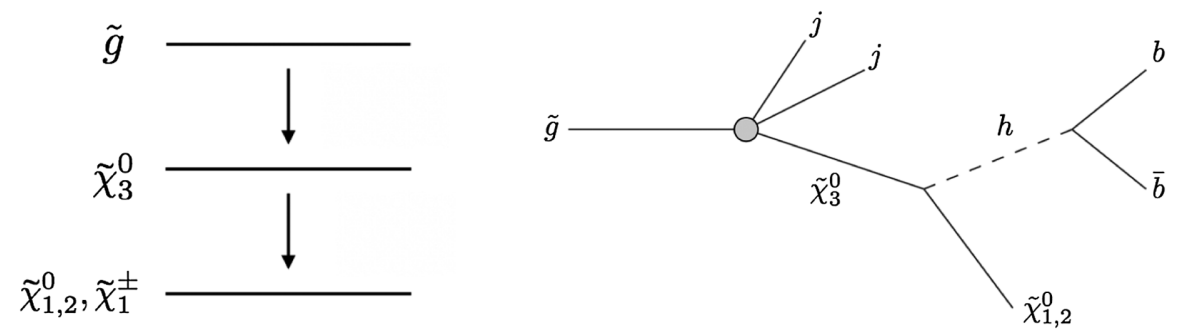

FIG. 1. Left: typical spectrum considered in the analysis with the decay channels shown close to the arrows. Right: gluino decay cascade involved in the signature of interest.

\section{THEORETICAL FRAMEWORK}

Identifying the lightest neutralino $\tilde{\chi}_{1}^{0}$ as the lightest supersymmetric particle (LSP), and thus a dark matter candidate in the presence of $R$ parity [10], is one of the most appealing features of the minimal supersymmetric extension of the Standard Model (MSSM) [11-13]. Given the strong LHC bounds on the mass of supersymmetric particles, and the plethora of null results on dark matter direct searches, one supersymmetric scenario that remains is an almost pure Higgsino with a mass $\sim 1.1 \mathrm{TeV}[14,15]$. This requirement (almost) fixes the theoretical framework in the electroweakino (neutralino/chargino) sector as it generically requires that $\mu \sim 1.1 \mathrm{TeV}$ (where $\mu$ is the super symmetric Higgsino mass) while $M_{1}, M_{2} \gg \mu$ (where $M_{1}$ and $M_{2}$ are soft supersymmetry breaking Majorana masses for the fermionic partners of the $U(1)_{Y}$ and $S U(2)$ gauge bosons, bino, and wino respectively).

The Majorana masses $M_{1,2}$ are defined at the low scale and their values depend on the mechanism of supersymmetry breaking. While the requirement of the Higgsino being the LSP rules out gauge mediation (for which the gravitino is the LSP) as the transmission mechanism for supersymmetry breaking, gravity mediation seems to be the preferred one, as there is room for the lightest neutralino to be the LSP and moreover the supersymmetric mass $\mu$ can be generated by the Giudice-Masiero mechanism [16]. In gravity mediation, all supersymmetry breaking parameters, and in particular $M_{1,2}$ are generated at the high (unification) scale, i.e., $M_{1,2}^{0}$, and their value at low scale is obtained by means of the renormalization group equation (RGE) running. Unification conditions are usually assumed, i.e., $M_{1}^{0}=M_{2}^{0}$, but even assuming that $M_{1}^{0} \sim M_{2}^{0}$, after the RGE running we have $M_{2} \sim 2 M_{1}$ so that the bino $\tilde{\chi}_{3}^{0}$ is lighter than the wino $\tilde{\chi}_{4}^{0}$. On the other hand we will deviate from the usual assumption of a common scalar mass at high scales in order to obtain the branching ratios discussed in the next paragraphs. ${ }^{1}$

Under these circumstances the neutralino sector is almost completely fixed: (i) There are two (almost) purely

\footnotetext{
${ }^{1}$ This situation can arise in the effective theory of some supergravity/superstring models [17].
}

Higgsinos, $\tilde{\chi}_{1}^{0}, \tilde{\chi}_{2}^{0}$, with masses $\sim 1.1 \mathrm{TeV}$ and a mass separation of a few $\mathrm{GeV}$. (ii) There is a bino $\tilde{\chi}_{3}^{0}$ with a mass $m_{\tilde{\chi}_{3}^{0}} \sim M_{1}$ and a wino with a mass $m_{\tilde{\chi}_{4}^{0}} \sim 2 m_{\tilde{\chi}_{3}^{0}}$. At the same time the constraints from the XENON1T experiment on direct detection [18], analyzed in Ref. [15], put the constraint, for the case of equal masses at the unification scale, $M_{1}^{0}=M_{2}^{0} \gtrsim 3.2 \mathrm{TeV}$, which translates into the lower bounds $m_{\tilde{\chi}_{3}^{0}} \gtrsim 1.5 \mathrm{TeV}$ and $m_{\tilde{\chi}_{4}^{0}} \gtrsim 2.7 \mathrm{TeV}$ [5]. As for the chargino sector, the lightest state $\tilde{\chi}_{1}^{ \pm}$is almost degenerate with the LSP, with a few GeV gap, while the heaviest chargino is almost degenerate with the heavy neutralino, so that $m_{\tilde{\chi}_{2}^{ \pm}} \gtrsim 2.7 \mathrm{TeV}$.

On the other hand the gluino $\tilde{g}$ mass $M_{\tilde{g}}$ is also fixed by the breaking mass $M_{3}^{0}$ at the unification scale. In our theoretical framework the gluino mass is not unified with the electroweak masses $M_{1,2}^{0}$ so that it will be considered as a free parameter. This is a safe assumption as the gluino mass does not enter the process of electroweak breaking at the tree level. We will assume that the gluino mass will be close to its present experimental bound $M_{\tilde{g}} \sim 2 \mathrm{TeV}$. Moreover we are going to assume, for simplicity, that all other sparticles including squarks are more massive than the gluino, nonetheless all decays are assumed to be prompt. In this case the possible channels for the gluino decay are $\tilde{g} \rightarrow \tilde{\chi}_{1,2}^{0} j j, \tilde{g} \rightarrow \tilde{\chi}_{1}^{ \pm} j j$, and $\tilde{g} \rightarrow \tilde{\chi}_{3}^{0} j j$, mediated by the decay $\tilde{g} \rightarrow \tilde{q}_{a}^{*} q_{a}$, where $a$ is a generation label, and followed by $\tilde{q}_{a}^{*} \rightarrow \tilde{\chi}_{1,2}^{0} q_{a}, \tilde{q}_{a}^{*} \rightarrow \tilde{\chi}_{1}^{ \pm} q_{a}$ (induced by the Yukawa coupling $y_{q_{a}}$ ) and $\tilde{q}_{a}^{*} \rightarrow \tilde{\chi}_{3}^{0} q_{a}$ (induced by the $U(1)$ gauge coupling $g_{1}$ ), respectively. The typical situation that current analyses consider and cover is that the direct decay to nearly degenerate Higgsinos dominates $\left(\tilde{\chi}_{1,2}^{0}, \tilde{\chi}_{1}^{ \pm}\right)$. If, instead, the gluino decays predominantly to $\tilde{\chi}_{3}^{0}$, one will get a final state with several energetic jets and $b$-quarks that will evade current bounds. The decay channels of the gluino depend on the details of the squark spectrum: if the first two generations of squark are less massive than the third generation, then the decay to $\tilde{\chi}_{3}^{0}$ is favored, being of electroweak nature as opposed to the decay to the Higgsino which is proportional to the corresponding Yukawa coupling. In Fig. 1 we have a schematic view of the spectrum and the gluino decay cascade that are going to be analyzed in the next section. 


\section{COLLIDER ANALYSIS}

The experimental signature under study at the LHC comes from the SUSY production of a pair of gluinos, $p p \rightarrow \tilde{g} \tilde{g}$, that decay into $\tilde{\chi}_{3}^{0}$ and two light jets $\left(\tilde{g} \rightarrow \tilde{\chi}_{3}^{0} j j\right)$. We consider then that each $\tilde{\chi}_{3}^{0}$ decays into the $\operatorname{LSP}\left(\tilde{\chi}_{1}^{0}\right)$ and the lightest MSSM Higgs boson, $h$, identified as the 125GeV SM-like Higgs boson discovered at the LHC, which decays into a pair of $b$-quarks. Therefore, the final state is made of four light jets, four $b$-jets, and a large amount of missing transverse energy $\left(4 j+4 b+E_{T}^{\text {miss }}\right)$, whose main SM backgrounds are QCD multijet; $Z+$ jets and $W+$ jets productions; $t \bar{t}$ production; $t \bar{t}$ production in association with electroweak or Higgs bosons, $t \bar{t}+X\left(X=W, Z, \gamma^{*}\right.$, $h$ ); and diboson production ( $W W, Z Z, W Z, W h$, and $Z h$ ) plus jets.

We develop our search strategy for a LHC center-ofmass energy of $\sqrt{s}=14 \mathrm{TeV}$ and a total integrated luminosity of $\mathcal{L}=1000 \mathrm{fb}^{-1}$, compatible with the highluminosity LHC (HL-LHC) phase. We make use of MADGRAPH_AMC@NLO2.8.1 [19] for the Monte Carlo generation of both signal and background events, whose parton shower and hadronization is performed with PYTHIA8.2 [20], while the detector response simulation is achieved with DELPHES3.3.3 [21]. From the proposed new physics signal, one would expect in the final state very energetic light jets and $b$-jets, coming from the decays of gluinos and Higgs bosons, respectively. Therefore, with the intention of reducing the large background cross sections and making event generation more efficient, we impose the following generator-level cuts on the $p_{T}$ of the light jets and $b$-jets for the background simulation ${ }^{2}$ :

$p_{T}^{j_{1}}>180 \mathrm{GeV}, \quad p_{T}^{j_{2}}>140 \mathrm{GeV}, \quad p_{T}^{j_{3}}>70 \mathrm{GeV}$,

$p_{T}^{j_{4}}>35 \mathrm{GeV}, \quad p_{T}^{b_{1}}>90 \mathrm{GeV}, \quad p_{T}^{b_{2}}>20 \mathrm{GeV}$,

$p_{T}^{b_{3}}>20 \mathrm{GeV}, \quad p_{T}^{b_{4}}>20 \mathrm{GeV}$,

where $j_{1} \ldots j_{4}\left(b_{1} \ldots b_{4}\right)$ runs from the most to the least energetic light $(b-)$ jet. Dealing with many jets in the final state, the MLM algorithm [22,23] was implemented for jet matching and merging. In order to optimize the simulation and checking that the jet related distributions are smooth, the xqcut was set to 20 for all simulated samples and qcut equal to 550, 50, and 30 for signal, $t \bar{t}$-like and backgrounds with bosons, respectively. Furthermore, we use a working point for the $b$-tagging efficiency of $\epsilon_{b}=$ $75 \%$ and misidentification-rate equal to 0.01 for light jets (0.1 for $c$-jets), as set by default in the ATLAS Delphes card. The simulation input files and the internal analysis codes are available upon request to the authors.

\footnotetext{
${ }^{2}$ For the signal simulation, we use the default cuts on the $p_{T}$ of the light jets and $b$-jets $\left(p_{T}^{j}>20 \mathrm{GeV}\right.$ and $\left.p_{T}^{b}>20 \mathrm{GeV}\right)$.
}

With this in mind, the following comments on the signal and backgrounds are pertinent:

(i) The SUSY spectrum and branching ratios for the signal benchmark have been computed with SOFTSUSY.4.1.10 [24-30], while the production cross section of a pair of gluinos is obtained from [31]. The relevant mass parameters of our benchmark for the proposed SUSY signature are $M_{\tilde{g}}=2.1 \mathrm{TeV}$, $m_{\tilde{\chi}_{3}^{0}}=1.6 \mathrm{TeV}$, and $m_{\tilde{\chi}_{1}^{0}}=1.2 \mathrm{TeV}$, with the first two generations of squarks at masses around $\sim 4 \mathrm{TeV}$ and the third generation of squarks decoupled. The corresponding gluino-pair production cross section and branching ratios are $\sigma(p p \rightarrow \tilde{g} \tilde{g})=1.1 \mathrm{fb}$, $\operatorname{BR}\left(\tilde{g} \rightarrow \tilde{\chi}_{3}^{0} j j\right)=0.82, \operatorname{BR}\left(\tilde{\chi}_{3}^{0} \rightarrow \tilde{\chi}_{1}^{0} h\right)=0.27$, and $\operatorname{BR}(h \rightarrow b \bar{b})=0.58$. Notice that the decay of neutralino $\tilde{\chi}_{3}^{0}$ to the other (almost degenerated) LSP is negligible. With these values, 20 signal events are expected for $\mathcal{L}=1000 \mathrm{fb}^{-1}$.

(ii) The QCD multijet background is unmanageable with our computational capacity, and is usually treated with data-driven techniques. In our case, taking into account that our signal will have a large amount of $E_{T}^{\text {miss }}$, variables related to this observable, such as the $E_{T}^{\text {miss }}$ significance, greatly reduce this class of backgrounds with instrumental missing transverse energy. Also, the characteristic spatial configuration of the missing transverse momentum can be exploited in order to reduce this background. We include an estimation of this background by recasting the analysis in [6], in which a similar final state is considered and a similar cut-based analysis is developed.

(iii) Regarding the $V+$ jets production, including both $Z+$ jets and $W+$ jets, we considered a pair of $b$-jets and a pair of light jets leading to four extra jets and a genuine source of missing energy through neutrinos coming from the decay of the gauge bosons (with $\mathrm{BR}(Z \rightarrow \nu \nu)=0.2 \quad$ and $\quad \mathrm{BR}(W \rightarrow l \nu)=0.21)$. Other combinations of extra jets do not have $b$ or light jets enough and more than 4 extra jets are out of our simulation capacity. Then, taking into account the generator setup, we expect $5.6 \times 10^{4}$ for $Z+$ jets and $3 \times 10^{5}$ events for $W+$ jets with $\mathcal{L}=1000 \mathrm{fb}^{-1}$.

(iv) Related to the $V+$ jets background, the diboson production can be safely neglected in this analysis since it is subdominant with an amount of roughly $10^{-3}$ times the $V+$ jets (which we will see it is already under control).

(v) The $t \bar{t}$ production, with both fully-hadronic and semileptonic decay channels, is the most dangerous background. The corresponding branching fractions are $\operatorname{BR}\left(t \bar{t}_{\text {had }}\right)=0.457$ and $\operatorname{BR}\left(t \bar{t}_{\text {semilep }}\right)=0.438$. After the generator-level cuts, we expect $1.36 \times 10^{6}$ and $0.42 \times 10^{6}$ events, respectively. We also 

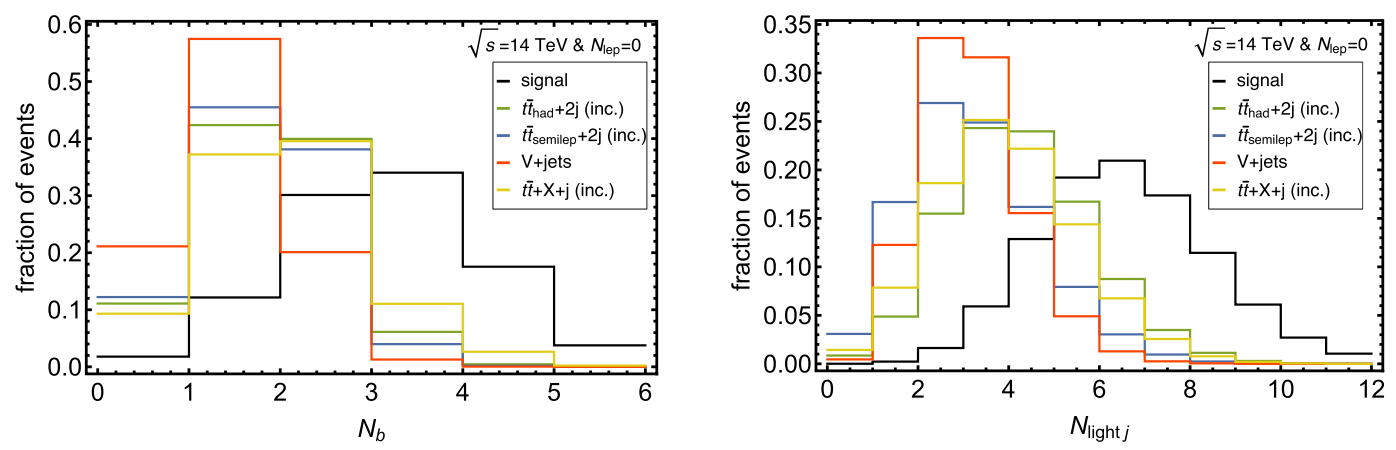

FIG. 2. Distributions (with a lepton veto) of the fraction of signal and background events of the number of identified $b$-jets $N_{b}$ (left panel) and the number of light jets $N_{j}$ (right panel).

consider one extra jet in the simulation, resulting in $0.83 \times 10^{6}$ and $0.25 \times 10^{6}$ events more for the hadronic and semileptonic channels, respectively. In addition, we include an estimation of $t \bar{t}+2 j$ taking into account an extra $10 \%$ factor to the simulated $t \bar{t}$ plus $t \bar{t}+j$ events (given by the ratio of the corresponding cross sections).

(vi) Concerning the $t \bar{t}+X$ backgrounds, even though is much smaller than the $t \bar{t}$ ones, the extra boson provide genuine source of missing energy (more $b$-jets) for the hadronic (semileptonic) top-quark pair. Explicitly, we consider $t \bar{t}_{\text {had }}+(Z \rightarrow \nu \nu), t \bar{t}_{\text {had }}+(W \rightarrow l \nu)$, $t \bar{t}_{\text {semilep }}+(Z \rightarrow b \bar{b}), \quad t \bar{t}_{\text {semilep }}+\left(\gamma^{*} \rightarrow b \bar{b}\right), \quad$ and $t \bar{t}_{\text {semilep }}+(h \rightarrow b \bar{b})$. We also include one extra jet to each process, leading to $2.9 \times 10^{3}$ expected events in this category.

Next we will perform a characterization of the signal against the dominant SM backgrounds in order to define the most promising signal regions for our search strategy. In our analysis, the previously defined backgrounds are separated in four categories: $t \bar{t}_{\text {had }}+2 j$ (inclusive), $t \bar{t}_{\text {semilep }}+2 j$ (inclusive), $V+$ jets, and $t \bar{t}+X+j$ (inclusive).

In Fig. 2 we depict the distributions of the fraction of signal and backgrounds events of the number of identified $b$-jets $N_{b}$ (left panel) and the number of light jets $N_{j}$ (right panel). In order to avoid one of the most dangerous background, the semileptonic $t \bar{t}$ production, we first set a lepton veto $\left(N_{\ell}=0\right)$, which have been already imposed on the distributions on both plots of Fig. 2. One of the most challenging task of the proposed signature is the identification of $b$-jets, since the signal is characterized by 4 bottom quarks coming from the Higgs boson decays. It is clear from the left panel of Fig. 2 that the requirement of identifying $4 b$-jets would reduce the number of signal events to less than half. Therefore, we are going to impose two class of selection cuts related to the number of identified $b$-jets: a loose cut with at least $2 b$-jets in the final state $\left(N_{b} \geq 2\right)$ and a tight cut, requiring at least 3 reconstructed $b$-jets $\left(N_{b} \geq 3\right)$. The signal consists also of 4 light jets, then we add to the selection-cut set the requisite of having at least 4 light jets in the final state $\left(N_{j} \geq 4\right)$. Thus, the selection cuts that characterize our signal are as follows:

$$
\begin{aligned}
& \text { loose: } N_{b} \geq 2, \quad N_{j} \geq 4, \quad N_{\ell}=0 \text {, } \\
& \text { tight: } N_{b} \geq 3, \quad N_{j} \geq 4, \quad N_{\ell}=0 \text {. }
\end{aligned}
$$

Figure 3 is devoted to the distributions, after the loose cut, of the fraction of signal and background events of six crucial kinematic variables: the transverse momentum of the leading $b$-jet $p_{T}^{b_{1}}$ (upper left panel); the transverse momentum of the leading light jet $p_{T}^{j_{1}}$ (upper right panel); the missing transverse energy $E_{T}^{\text {miss }}$ (medium left panel); the azimuthal angle difference $\Delta \phi\left(j_{1}, \vec{p}_{T}^{\text {miss }}\right)$ (medium right panel), defined as angular separation between the leading jet and the missing transverse momentum; the $E_{T}^{\text {miss }}$ significance (lower left panel), which is the ratio of the missing transverse energy over the square root of the hadronic activity $\left(E_{T}^{\text {miss }} / \sqrt{H_{T}}\right)$ for which we consider the scalar sum of the transverse momentum of all the jets $\left(H_{T}=\sum_{\text {all } b, j} p_{T}\right)$; and the effective mass $m_{\text {eff }}$ (lower right panel), defined as the sum of the missing transverse energy plus the hadronic activity $\left(m_{\text {eff }}=E_{T}^{\text {miss }}+H_{T}\right)$.

Some comments are in order. The $p_{T}^{b_{1}}$ distributions for the background events have their maximum around $100 \mathrm{GeV}$, with a sharp drop after that, while the $p_{T}^{b_{1}}$ distribution for the signal is less choppy, with its maximum around $500 \mathrm{GeV}$. Recall also here that the simulation of the backgrounds has been performed with the generator-level cuts, while the signal events have been simulated with only the default cuts. Therefore, a severe cut on $p_{T}^{b_{1}}$ will help to greatly reduce the background events, without affecting the signal events too much.

On the other hand, a priori no similar conclusion can be drawn about the $p_{T}^{j_{1}}$ distributions of the backgrounds, which mimic the signal distribution very well. However, 

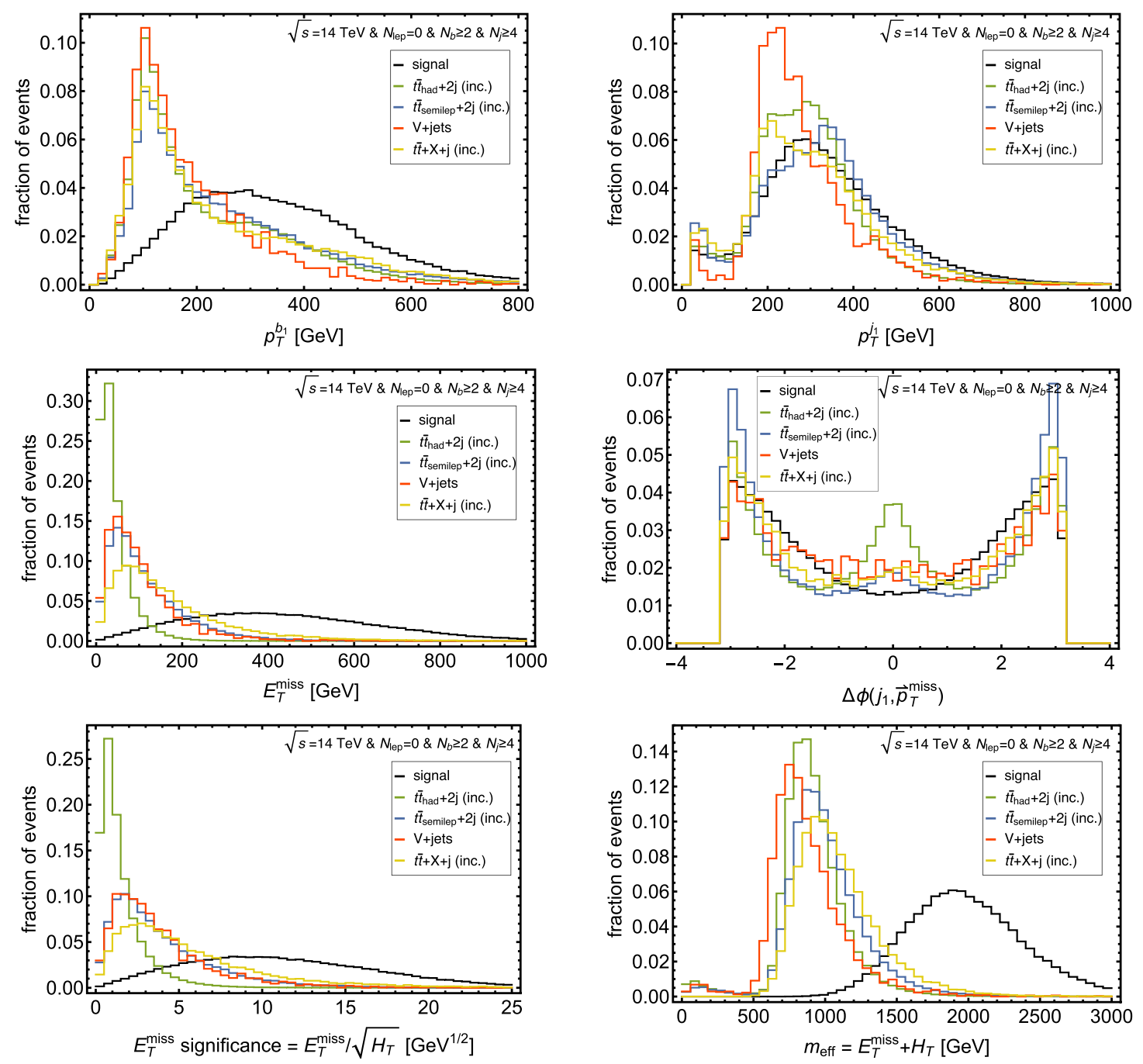

FIG. 3. Distributions (with a lepton veto, at least $2 b$-jets and 4 light jets) of the fraction of signal and background events of the transverse momentum of leading $b$-jet $p_{T}^{b_{1}}$ (upper left panel), the transverse momentum of the leading light jet $p_{T}^{j_{1}}$ (upper right panel), the missing transverse energy $E_{T}^{\text {miss }}$ (medium left panel), the azimuthal angle difference $\Delta \phi\left(j_{1}, \vec{p}_{T}^{\text {miss }}\right)$ between the leading jet and the $\vec{p}_{T}^{\text {miss }}$ (medium right panel), the $E_{T}^{\text {miss }}$ significance (lower left panel), and the effective mass $m_{\text {eff }}$ (lower right panel).

we will see later when we define our search strategy, that the cuts on the $p_{T}$ of the four leading light jets remove a large number of background events.

The $E_{T}^{\text {miss }}$ distribution for the signal is practically flat (in the range from $200 \mathrm{GeV}$ to $600 \mathrm{GeV}$, more or less), while for the backgrounds it peaks below $100 \mathrm{GeV}$ and drops sharply thereafter, with very little fraction of events above $200 \mathrm{GeV}$. It is therefore to be expected that a cut around this value eliminates much of the background events without much change in the number of signal events.

In addition, $E_{T}^{\text {miss }}$ significance distributions for the backgrounds are mostly below $5 \mathrm{GeV}^{1 / 2}$, with peaks around values of $2-3 \mathrm{GeV}^{1 / 2}$. The signal distribution, however, is much less steep, being more or less flat between 5 and $15 \mathrm{GeV}^{1 / 2}$. From this we can also conclude that a $E_{T}^{\text {miss }}$ significance cut above $5 \mathrm{GeV}^{1 / 2}$ should be very helpful in reducing the backgrounds without affecting the signal. On the other hand, a cut $\left|\Delta \phi\left(j_{1}, \vec{p}_{T}^{\text {miss }}\right)\right|>0.4$ suppress a fraction of $t \bar{t}_{\text {had }}$ production and also reject a large fraction of the QCD multijet (where the instrumental missing energy comes from jet energy mismeasurements and neutrinos inside the hadrons). Therefore, we will consider 0.7 events at the end of our cut-based analysis following [6] for $\mathcal{L}=1000 \mathrm{fb}^{-1}$.

Finally, the effective mass $m_{\text {eff }}$ also appears to be a very efficient variable for separating signal from background. The signal distribution peaks around $1800 \mathrm{GeV}$ while the background ones have peaks around $700-800 \mathrm{GeV}$, with very few events beyond $1400 \mathrm{GeV}$.

All these six kinematic variables, shown in Fig. 3, together with the transverse momenta of the subleading light jets and $b$-jets, not shown here for space saving, 
indicate in general a very distinct behavior between signal and background. The distributions after the tight cut are very similar to the previous ones, and are also not shown here for space saving. However we will see that the $m_{\text {eff }}$ cut can be relaxed by demanding at least three $b$ tagged jets and $E_{T}^{\text {miss }}>150 \mathrm{GeV}$. This motivates the definition of our search strategy, through the cuts shown below, separating into two signal regions: a first signal region (SR1) in which we ask for at least two $b$-jets in the final state and another one (SR2) with at least three reconstructed $b$-jets. Also, both signal regions require at least four light jets. The $p_{T}$ cuts at detector level for all the jets are then:

$$
\begin{array}{ll}
p_{T}^{j_{1}}>200 \mathrm{GeV}, & p_{T}^{j_{2}}>150 \mathrm{GeV}, \\
p_{T}^{j_{3}}>80 \mathrm{GeV}, & p_{T}^{j_{4}}>40 \mathrm{GeV},
\end{array}
$$

loose: $p_{T}^{b_{1}}>100 \mathrm{GeV}, \quad p_{T}^{b_{2}}>60 \mathrm{GeV}$,

$$
\text { tight: } p_{T}^{b_{1}}>100 \mathrm{GeV}, \quad p_{T}^{b_{2}}>60 \mathrm{GeV}
$$$$
p_{T}^{b_{3}}>35 \mathrm{GeV} \text {. }
$$

Based on the above, we define the SR1 search strategy with the following cuts:

(i) Loose selection cuts of Eq. (2), (ii) loose $p_{T}$ cuts of Eq. (3),

(iii) MET cuts given by $E_{T}^{\text {miss }}>150 \mathrm{GeV}$, $\left|\Delta \phi\left(j_{1}, \vec{p}_{T}^{\text {miss }}\right)\right|>0.4$

(iv) and $m_{\text {eff }}>1800 \mathrm{GeV}$,

while the SR2 search strategy has these cuts:

(i) Tight selection cuts of Eq. (2),

(ii) tight $p_{T}$ cuts of Eq. (3),

(iii) MET cuts given by $E_{T}^{\text {miss }}>150 \mathrm{GeV}$, $\left|\Delta \phi\left(j_{1}, \vec{p}_{T}^{\text {miss }}\right)\right|>0.4$

(iv) and $m_{\text {eff }}>1400 \mathrm{GeV}$.

In order to study the potential of our search strategies, we are going to make use of the following expression for the statistical significance of the number of signal events, $S$, with respect to the number of background events, $B[32,33]$ :

$$
\mathcal{S}_{\text {sta }}=\sqrt{-2\left((B+S) \log \left(\frac{B}{B+S}\right)+S\right)}
$$

In addition, to obtain a more realistic estimate of the significances, ${ }^{3}$ we can take background systematic uncertainties into account by modifying Eq. (4) as follows $[32,33]$ :

$$
\mathcal{S}_{\text {sys }}=\sqrt{2\left((B+S) \log \left(\frac{(S+B)\left(B+\sigma_{B}^{2}\right)}{B^{2}+(S+B) \sigma_{B}^{2}}\right)-\frac{B^{2}}{\sigma_{B}^{2}} \log \left(1+\frac{\sigma_{B}^{2} S}{B\left(B+\sigma_{B}^{2}\right)}\right)\right)},
$$

where $\sigma_{B}=(\Delta B) B$, with $\Delta B$ being the relative systematic uncertainty, that we choose to be, in a conservative way, of $30 \%$. In particular, this value includes the uncertainty associated to the limited statistics of our analysis, in which we mostly suppress the expected backgrounds, as we will see in the following.

We are now in a position to apply our search strategies on the events of our signal and the backgrounds generated for an LHC energy of $14 \mathrm{TeV}$ and a total integrated luminosity of $1000 \mathrm{fb}^{-1}$. In Tabs. I and II the cut flow of the SR1 and SR2 signal regions are shown, respectively, together with their corresponding significances as we apply each of the cuts. Notice that QCD multijet estimation ( 0.7 events) is included in the significances at the end of the search strategy for each SR, as discussed previously.

In the SR1 case (Table I), we see that the selection cuts reduce more than one order the magnitude all the background events, while keeping the $75 \%$ of the signal events.

\footnotetext{
${ }^{3}$ Using the ZSTATS package [34], we have verified that the significances obtained with Eqs. (4) and (5) are compatible with the values obtained with the expressions for discovery significances proposed in $[35,36]$, with differences of at most $5 \%$.
}

In this signal region, the loose $p_{T}$ cuts are very efficient, reducing backgrounds by more than two orders of magnitude and only half the signal. The $E_{T}^{\text {miss }}$ cut is also very useful, eliminating most of the $t \bar{t}$ and $t \bar{t}+X$ events and bringing the $V+$ jets background to zero, while barely affecting the signal events. Finally, the $m_{\text {eff }}$ variable eliminates most of the $t \bar{t}$-like events, leaving only 2.5 events of the total $t \bar{t}$ background and keeping 5.1 signal events, more than $25 \%$ of those initially expected. This all adds up to a final statistical significance somewhat greater than $2 \sigma$, even if we consider a conservative $30 \%$ systematic uncertainties in the background.

The results for the SR2 search strategy are more stimulating, as shown in the cut flow of Table II. The tight selection cuts reduce the hadronic $t \bar{t}$ background by two orders of magnitude and all other backgrounds by more than three orders of magnitude, while keeping half of the signal events. The $p_{T}$ cuts eliminate the $V+$ jets background and again reduce the remaining backgrounds by more than two orders of magnitude, with half of the remaining signal events surviving. The $E_{T}^{\text {miss }}$ cut again hardly affects the signal, reduces by two orders of magnitude the events of the hadronic $t \bar{t}$ background, which are 
TABLE I. Cut flow for SR1 with $\mathcal{L}=1000 \mathrm{fb}^{-1}$. Loose selection cuts shown in Eq. (2) and $p_{T}$ cuts of Eq. (3). Significances from Eqs. (4) and (5), the latter with a background systematic uncertainty of 30\%. A QCD multijet estimation of 0.7 events [6] is included in the significances of the last step.

\begin{tabular}{|c|c|c|c|c|c|c|c|}
\hline Process & Signal & $t \bar{t}_{\text {had }}+2 j$ (inc.) & $t \bar{t}_{\text {semilep }}+2 j$ (inc.) & $V+$ jets & $t \bar{t} X+j$ (inc.) & $\mathcal{S}_{\text {sta }}$ & $\mathcal{S}_{\text {sys }}$ \\
\hline Expected & 20 & $2.4 \times 10^{6}$ & $0.74 \times 10^{6}$ & $3.56 \times 10^{5}$ & $2.9 \times 10^{3}$ & 0.01 & $2 \times 10^{-5}$ \\
\hline Selection cut & 15.7 & $3.28 \times 10^{5}$ & $2.8 \times 10^{4}$ & 4435 & 505.5 & 0.03 & $1.5 \times 10^{-4}$ \\
\hline Loose $p_{T}$ cuts & 7.7 & 8075 & 285.2 & 12.7 & 14.3 & 0.09 & $3.3 \times 10^{-3}$ \\
\hline MET cuts & 6.6 & 76.6 & 33.3 & 0 & 4.6 & 0.61 & 0.18 \\
\hline$m_{\mathrm{eff}}>1800 \mathrm{GeV}$ & 5.1 & 1.0 & 1.3 & 0 & 0.2 & 2.37 & 2.01 \\
\hline
\end{tabular}

TABLE II. Cut flow for SR2 with $\mathcal{L}=1000 \mathrm{fb}^{-1}$. Tight selection cuts shown in Eq. (2) and $p_{T}$ cuts of Eq. (3). Significances from Eqs. (4) and (5), the latter with a background systematic uncertainty of 30\%. A QCD multijet estimation of 0.7 events [6] is included in the significances of the last step.

\begin{tabular}{|c|c|c|c|c|c|c|c|}
\hline Process & Signal & $t \bar{t}_{\text {had }}+2 j$ (inc.) & $t \bar{t}_{\text {semilep }}+2 j$ (inc.) & $V+$ jets & $t \bar{t} X+j$ (inc.) & $\mathcal{S}_{\text {sta }}$ & $\mathcal{S}_{\text {sys }}$ \\
\hline Expected & 20 & $2.19 \times 10^{6}$ & $0.67 \times 10^{6}$ & $3.56 \times 10^{5}$ & $2.9 \times 10^{3}$ & 0.01 & $2 \times 10^{-5}$ \\
\hline Selection cut & 9.8 & $3.06 \times 10^{4}$ & 2025 & 145.7 & 94.1 & 0.06 & $1 \times 10^{-3}$ \\
\hline Tight $p_{T}$ cuts & 4.4 & 216.7 & 4.1 & 0 & 2.1 & 0.29 & 0.06 \\
\hline MET cuts & 3.9 & 2.1 & 0.7 & 0 & 0.4 & 1.87 & 1.59 \\
\hline$m_{\mathrm{eff}}>1400 \mathrm{GeV}$ & 3.8 & 0 & 0.3 & 0 & 0 & 2.73 & 2.52 \\
\hline
\end{tabular}

finally removed by the $m_{\text {eff }}$ cut, which hardly modifies the signal, eliminates the $t \bar{t}+X$ events and leaves the only surviving background in this signal region, semileptonic $t \bar{t}$, at 0.36 events. In the end, in this signal region we obtain for both significance estimates values close to the evidence level. At this point, it is important to note that in both signal regions the cuts can be further adjusted, preserving at least three signal events and killing all the simulated backgrounds at the same time. For instance, for SR1 (SR2) with $m_{\text {eff }}>2100 \mathrm{GeV}\left(m_{\text {eff }}>1500 \mathrm{GeV}\right), 3.2$ (3.7) signal events remain and the background events vanish. Notice that this kinematic variable summarizes the main feature of our signal, with several energetic light jets and $b$-jets, that differs from the more conventional ones (with full decays to the LSP).

The projections for a luminosity of $3000 \mathrm{fb}^{-1}$, considering that the number of signal and background events increase in the same way, are very promising. For the SR1 search strategy we obtain $\mathcal{S}_{\text {sta }}=4.12$ and $\mathcal{S}_{\text {sys }}=2.76$, and $\mathcal{S}_{\text {sta }}=4.73$ and $\mathcal{S}_{\text {sys }}=3.97$ for the SR2 case. That is, for the future high-luminosity phase of the LHC, one could expect statistical significances above the evidence level in the SR1 signal region and very near the discovery level in the SR2. If we consider a $30 \%$ systematic uncertainties in the background, the significances degrade for both signal regions, obtaining values slightly below the level of evidence for SR1 and almost 4 standard deviations for SR2. This uncertainty value is very conservative for the last HL-LHC upgrade but it shows the robustness of our search strategy.
In these MSSM scenarios, we have fixed $M_{\tilde{\chi}_{1}^{0}}=$ $1.15 \mathrm{TeV}$, in order to accomplish with the cosmological and dark matter relic density requirements, as explained in [5]. However, it is interesting and very illustrative to study the potential of our search strategy if we apply it to other benchmarks in which the other two relevant parameters of these scenarios, $M_{\tilde{g}}$ and $M_{\tilde{\chi}^{0}}$, are varied. In Fig. 4 we display the contour lines of $\mathcal{S}_{\text {sys }}$ in the plane $\left[M_{\tilde{g}}, M_{\tilde{\chi}_{3}^{0}}\right]$ for both signal regions SR1 (left panel) and SR2 (right panel). The red, blue and green lines correspond to values of $\mathcal{S}_{\text {sys }}$ of $2 \sigma, 3 \sigma$, and $5 \sigma$, respectively (with a systematic uncertainty of $30 \%$ in the background), considering total integrated luminosities of $\mathcal{L}=1000 \mathrm{fb}^{-1}$ (solid lines) and $\mathcal{L}=3000 \mathrm{fb}^{-1}$ (dotted lines). For the SR1 signal region, with $\mathcal{L}=1000 \mathrm{fb}^{-1}$, we obtain at most $2 \sigma$ significances, for gluino mass values below about $2.15 \mathrm{TeV}$ and bino masses below $1.5 \mathrm{TeV}$, although in some cases the latter can be up to $1.6 \mathrm{TeV}$. The projections for $3000 \mathrm{fb}^{-1}$ allow us to reach $2 \sigma$ significances for all $M_{\tilde{g}}$ values in our parameter space, except when the bino mass exceeds the range of 1.55-1.65 TeV. Likewise, evidence-level significances can be obtained for $M_{\tilde{g}}$ values below $2.1 \mathrm{TeV}$ and bino masses below about $1.5 \mathrm{TeV}$. The results for SR2 are much more promising, even at $1000 \mathrm{fb}^{-1}$ luminosity, for which we obtain $2 \sigma$ signatures over the entire parameter space except when $M_{\tilde{\chi}_{3}^{0}}$ starts to be larger than $1.6 \mathrm{TeV}$ and $M_{\tilde{g}}$ is smaller than $2.15 \mathrm{TeV}$. For this same luminosity, our 

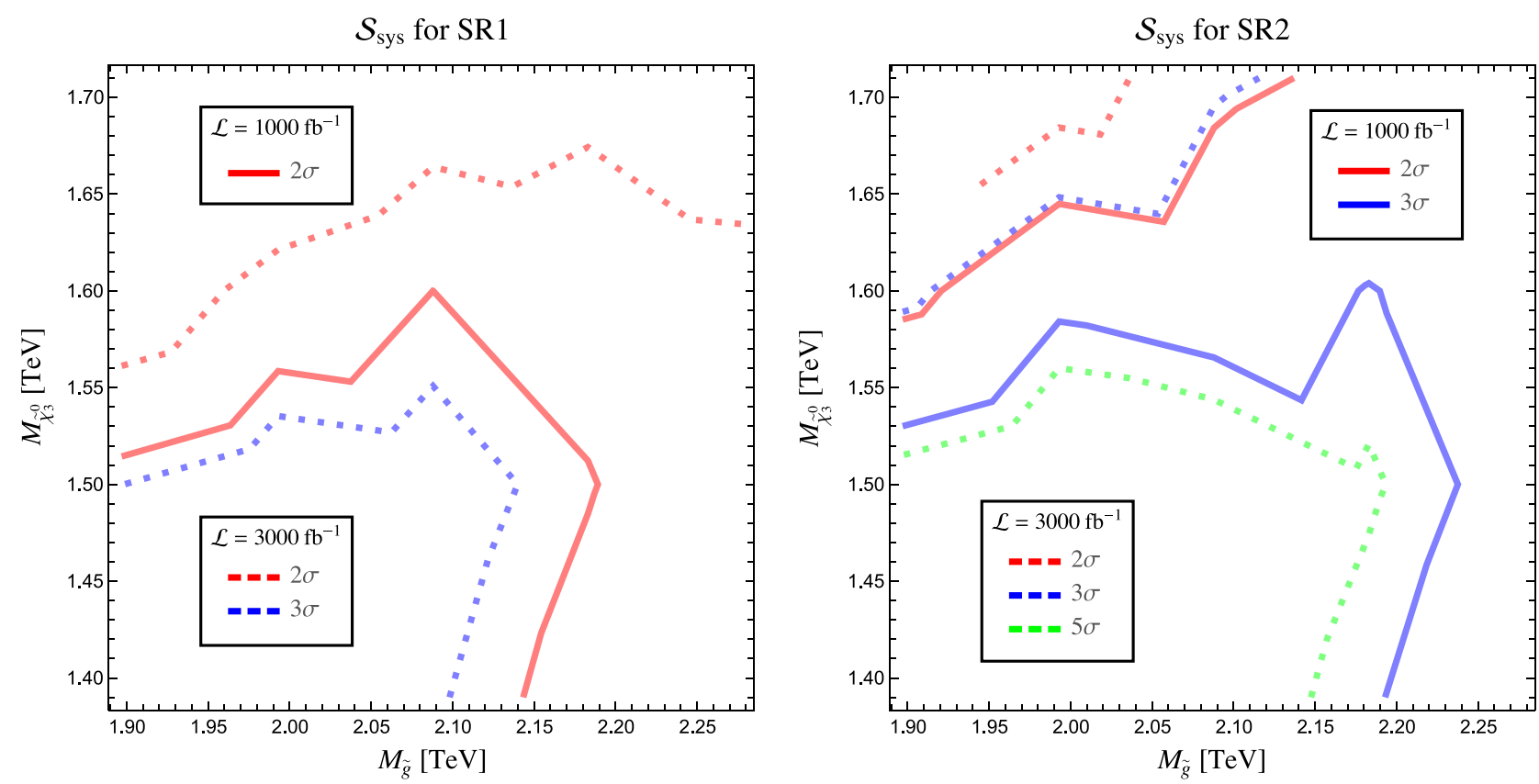

FIG. 4. Contour plots for both SR1 (left) and SR2 (right) in the plane $\left[M_{\tilde{g}}, M_{\tilde{\chi}_{3}^{0}}\right]$ ). The red, blue and green colors are the $\mathcal{S}_{\text {sys }}$ (systematic uncertainty of $30 \%$ ) with values $2 \sigma, 3 \sigma$ and $5 \sigma$, respectively. Solid(dotted) lines correspond to $\mathcal{L}=1000(3000) \mathrm{fb}^{-1}$.

search strategy allows us to obtain evidence-level significances as long as $M_{\tilde{g}}$ is less than about $2.2 \mathrm{TeV}$ and $M_{\tilde{\chi}_{3}^{0}}$ does not exceed $1.6 \mathrm{TeV}$. The results for this signal region with $3000 \mathrm{fb}^{-1}$ are much more encouraging. $2 \sigma$ significances are obtained in almost all the parameter space, except for a small region in the upper left corner of the plane $\left[M_{\tilde{g}}, M_{\tilde{\chi}_{3}^{0}}\right]$, with bino masses greater than $1.65 \mathrm{TeV}$ and gluino masses less than $2.05 \mathrm{TeV}$. The contour line for significances at the evidence level practically coincides with that of $2 \sigma$ with $\mathcal{L}=1000 \mathrm{fb}^{-1}$. Finally, our search strategy results in significances at the discovery level for an important area of the plane $\left[M_{\tilde{g}}\right.$, $M_{\tilde{\chi}_{3}^{0}}$, with $M_{\tilde{g}} \lesssim 2.15 \mathrm{TeV}$ and $M_{\tilde{\chi}_{3}^{0}} \lesssim 1.5 \mathrm{TeV}$.

These results show, on the one hand, the robustness of our search strategy, and on the other hand, its applicability to other supersymmetric spectra of the MSSM scenarios we work with, beyond the benchmark for which it was optimized. Within the range of $M_{\tilde{g}}$ and $M_{\tilde{\chi}_{3}^{0}}$ values considered, we are able to obtain significances up to the evidence level in SR1 and at the discovery level in SR2. These results motivate that this class of MSSM scenarios deserve special attention and dedicated interpretations by the LHC experiments.

\section{CONCLUSIONS}

In this work we have developed a proof-of-con cept collider analysis at the HL-LHC for a new SUSY signal (whose spectrum evades current LHC searches): $p p \rightarrow \tilde{g} \tilde{g} \rightarrow\left(\tilde{\chi}_{3}^{0} j j\right)\left(\tilde{\chi}_{3}^{0} j j\right) \rightarrow\left(\tilde{\chi}_{1}^{0} h j j\right)\left(\tilde{\chi}_{1}^{0} h j j\right) \rightarrow 4 j+4 b+$ $E_{T}^{\text {miss }}$. The more problematic SM backgrounds of this experimental signature are $t \bar{t}, V+$ jets $(V=W, Z)$, and $t \bar{t}+X\left(X=W, Z, \gamma^{*}, h\right)$, which all turn out to be under control after the cuts of our search strategy. The selection cuts define two signal regions, SR1 with $N_{b} \geq 2$ and SR2 with $N_{b} \geq 3$, to which we subsequently applied cuts on the most relevant kinematic variables: the transverse momenta of light and $b$-jets, $E_{T}^{\text {miss }}$, and $m_{\text {eff }}$, which is the sum of $E_{T}^{\text {miss }}$ plus the hadronic activity, $H_{T}$. With a center-of-mass energy of $14 \mathrm{TeV}$ and a total integrated luminosity of $1000 \mathrm{fb}^{-1}$, for the considered range of $M_{\tilde{g}}$ (1.9-2.3 TeV) and $M_{\tilde{\chi}_{3}^{0}}(1.4-1.7 \mathrm{TeV})$, we reach signal significances around $2 \sigma$ for SR1 and at the evidence level $(3 \sigma)$ for SR2. The prospects for $3000 \mathrm{fb}^{-1}$ are very encouraging, with significances of $3 \sigma$ for SR1 and up to the discovery level $(5 \sigma)$ for SR2, indicating that this novel interpretation deserves the development of dedicated analyses by the LHC experiments.

\section{ACKNOWLEDGMENTS}

The work of E. A. and R. M. is partially supported by the "Atracción de Talento" program (Modalidad 1) of the Comunidad de Madrid (Spain) under the Grant No. 2019-T1/TIC-14019 and by the Spanish Research Agency (Agencia Estatal de Investigación) through the grant IFT Centro de Excelencia Severo Ochoa 
No. SEV-2016-0597 (E. A., R. M.). The work of E. A. is also partially supported by CONICET and ANPCyT under Projects No. PICT 2016-0164, No. PICT 2017-2751, and No. PICT 2017-2765. The work of A. D. was partially supported by the National Science Foundation under Grant No. PHY-1820860. The work of M. Q. is partly supported by Spanish MINEICO under Grant No. FPA 2017-88915-P, by the Catalan Government under Grant No. 2017SGR1069, and by Severo Ochoa Excellence Program of MINEICO under Grant No. SEV-2016-0588. IFAE is partially funded by the CERCA program of the Generalitat de Catalunya.
[1] ATLAS Collaboration, Observation of a new particle in the search for the Standard Model Higgs boson with the ATLAS detector at the LHC, Phys. Lett. B 716, 1 (2012).

[2] CMS Collaboration, Observation of a new boson at a mass of $125 \mathrm{GeV}$ with the CMS Experiment at the LHC, Phys. Lett. B 716, 30 (2012).

[3] S. Norberg, Searches for strong supersymmetry, Moriond EW 2021, http://moriond.in2p3.fr/2021/EW/slides/2_bsm _04_norberg.pdf (2021).

[4] A. Elliot and Strong SUSY at ATLAS and CMS, Moriond QCD 2021, http://moriond.in2p3.fr/QCD/2021/Wednesday Morning/Elliot.pdf (2021).

[5] A. Delgado and M. Quirós, Higgsino dark matter in the MSSM, Phys. Rev. D 103, 015024 (2021).

[6] ATLAS Collaboration, Search for squarks and gluinos in final states with jets and missing transverse momentum using $139 \mathrm{fb}^{-1}$ of $\sqrt{s}=13 \mathrm{TeV} p p$ collision data with the ATLAS detector, J. High Energy Phys. 02 (2021) 143.

[7] ATLAS Collaboration, Search for supersymmetry in final states with missing transverse momentum and multiple $b$-jets in proton-proton collisions at $\sqrt{s}=13 \mathrm{TeV}$ with the ATLAS detector, J. High Energy Phys. 06 (2018) 107.

[8] ATLAS Collaboration, Search for new phenomena in final states with large jet multiplicities and missing transverse momentum using $\sqrt{s}=13 \mathrm{TeV}$ proton-proton collisions recorded by ATLAS in Run 2 of the LHC, J. High Energy Phys. 10 (2020) 062.

[9] CMS Collaboration, Search for supersymmetry in protonproton collisions at $13 \mathrm{TeV}$ in final states with jets and missing transverse momentum, J. High Energy Phys. 10 (2019) 244.

[10] G. Jungman, M. Kamionkowski, and K. Griest, Supersymmetric dark matter, Phys. Rep. 267, 195 (1996).

[11] H. P. Nilles, Supersymmetry, supergravity and particle physics, Phys. Rep. 110, 1 (1984).

[12] H. E. Haber and G. L. Kane, The search for supersymmetry: Probing physics beyond the Standard Model, Phys. Rep. 117, 75 (1985).

[13] J. Gunion and H. E. Haber, Higgs bosons in supersymmetric models. 1., Nucl. Phys. B272, 1 (1986).

[14] L. Roszkowski, E. M. Sessolo, and S. Trojanowski, WIMP dark matter candidates and searches-current status and future prospects, Rep. Prog. Phys. 81, 066201 (2018).

[15] K. Kowalska and E. M. Sessolo, The discreet charm of higgsino dark matter-A pocket review, Adv. High Energy Phys. 2018, 6828560 (2018).
[16] G. F. Giudice and A. Masiero, A natural solution to the mu problem in supergravity theories, Phys. Lett. B 206, 480 (1988).

[17] A. Brignole, L. E. Ibáñez, and C. Muñoz, Soft supersymmetry breaking terms from supergravity and superstring models, Adv. Ser. Dir. High Energy Phys. 21, 244 (2010).

[18] XENON Collaboration, Dark Matter Search Results from a One Ton-Year Exposure of XENON1T, Phys. Rev. Lett. 121, 111302 (2018).

[19] J. Alwall, R. Frederix, S. Frixione, V. Hirschi, F. Maltoni, O. Mattelaer, H.-S. Shao, T. Stelzer, P. Torrielli, and M. Zaro, The automated computation of tree-level and nextto-leading order differential cross sections, and their matching to parton shower simulations, J. High Energy Phys. 07 (2014) 079.

[20] T. Sjstrand, S. Ask, J. R. Christiansen, R. Corke, N. Desai, P. Ilten, S. Mrenna, S. Prestel, C. O. Rasmussen, and P. Z. Skands, An introduction to PYTHIA8.2, Comput. Phys. Commun. 191, 159 (2015).

[21] DELPHES 3 Collaboration, DELPHES 3, A modular framework for fast simulation of a generic collider experiment, J. High Energy Phys. 02 (2014) 057.

[22] M. Mangano, The so-called MLM prescription for ME/PS matching, Fermilab ME/MC Tuning Workshop, 4, 2002, http://www-cpd.fnal.gov/personal/mrenna/tuning/nov2002/ mlm.pdf.gz (2002).

[23] M. L. Mangano, M. Moretti, F. Piccinini, and M. Treccani, Matching matrix elements and shower evolution for topquark production in hadronic collisions, J. High Energy Phys. 01 (2007) 013.

[24] B. C. Allanach, SOFTSUSY: A program for calculating supersymmetric spectra, Comput. Phys. Commun. 143, 305 (2002).

[25] B. C. Allanach and T. Cridge, The calculation of sparticle and Higgs decays in the minimal and next-to-minimal supersymmetric Standard Models: SOFTSUSY4.0, Comput. Phys. Commun. 220, 417 (2017).

[26] B. C. Allanach, P. Athron, L. C. Tunstall, A. Voigt, and A. G. Williams, Next-to-minimal SOFTSUSY, Comput. Phys. Commun. 185, 2322 (2014).

[27] B. C. Allanach and M. A. Bernhardt, Including R-parity violation in the numerical computation of the spectrum of the minimal supersymmetric standard model: SOFTSUSY, Comput. Phys. Commun. 181, 232 (2010).

[28] B. C. Allanach, C. H. Kom, and M. Hanussek, Computation of neutrino masses in r-parity violating supersymmetry: SOFTSUSY3.2, Comput. Phys. Commun. 183, 785 (2012). 
[29] B. C. Allanach, A. Bednyakov, and R. Ruiz de Austri, Higher order corrections and unification in the minimal supersymmetric Standard Model: SOFTSUSY3.5, Comput. Phys. Commun. 189, 192 (2015).

[30] B. C. Allanach, S. P. Martin, D. G. Robertson, and R. R. de Austri, The inclusion of two-loop SUSYQCD corrections to gluino and squark pole masses in the minimal and next-to-minimal supersymmetric Standard Model: SOFTSUSY3.7, Comput. Phys. Commun. 219, 339 (2017).

[31] C. Borschensky, Z. Gecse, M. Kraemer, R. van der Leeuw, A. Kulesza, M. Mangano et al., LHC SUSY Cross Section Working Group, https://twiki.cern.ch/twiki/bin/view/LHC Physics/SUSYCrossSections, 2020.
[32] G. Cowan, K. Cranmer, E. Gross, and O. Vitells, Asymptotic formulae for likelihood-based tests of new physics, Eur. Phys. J. C 71, 1554 (2011).

[33] G. Cowan, Discovery sensitivity for a counting experiment with background uncertainty, Tech. Rep., Royal Holloway, London, 2012, http://www.pp.rhul.ac.uk/ cowan/stat/ medsig/medsigNote.pdf (2012).

[34] P. N. Bhattiprolu, S. P. Martin, and J. D. Wells, Zstats package, https://github.com/prudhvibhattiprolu/Zstats/, 2020.

[35] N. Kumar and S. P. Martin, Vectorlike leptons at the large hadron collider, Phys. Rev. D 92, 115018 (2015).

[36] P. N. Bhattiprolu, S. P. Martin, and J. D. Wells, Criteria for projected discovery and exclusion sensitivities of counting experiments, Eur. Phys. J. C 81, 123 (2021). 